\title{
Electrically driven single-electron spin resonance in a slanting Zeeman field
}

\author{
M. PIORO-LADRIÈRE ${ }^{1 *}$, T. OBATA ${ }^{1}$, Y. TOKURA ${ }^{1,2}$, Y.-S. SHIN ${ }^{1}$, T. KUBO $^{1}$, K. YOSHIDA ${ }^{1}$, T. TANIYAMA ${ }^{3,4}$ \\ AND S. TARUCHA ${ }^{1,5,6}$
}

\author{
${ }^{1}$ Quantum Spin Information Project, ICORP, Japan Science and Technology Agency, Atsugi-shi, 243-0198, Japan \\ ${ }^{2}$ NTT Basic Research Laboratories, NTT Corporation, Atsugi-shi, 243-0198, Japan \\ ${ }^{3}$ Materials and Structures Laboratory, Tokyo Institute of Technology, 4259 Nagatsuta, Yokohama, 226-8503, Japan \\ ${ }^{4}$ PRESTO, Japan Science and Technology Agency, 4-1-8 Honcho Kawaguchi, Saitama 332-0012, Japan \\ ${ }^{5}$ Department of Applied Physics, University of Tokyo, Hongo, Bunkyo-ku, Tokyo, 113-8656, Japan \\ ${ }^{6}$ Institute for Nano Quantum Information Electronics, University of Tokyo, Komaba, Meguro-ku, Tokyo 153-8505, Japan \\ *e-mail: michel@tarucha.jst.go.jp
}

The rapid rise of spintronics and quantum information science has led to a strong interest in developing the ability to coherently manipulate electron spins ${ }^{1}$. Electron spin resonance $^{2}$ is a powerful technique for manipulating spins that is commonly achieved by applying an oscillating magnetic field. However, the technique has proven very challenging when addressing individual spins ${ }^{3-5}$. In contrast, by mixing the spin and charge degrees of freedom in a controlled way through engineered non-uniform magnetic fields, electron spin can be manipulated electrically without the need of high-frequency magnetic fields $s^{6,7}$. Here we report experiments in which electrically driven addressable spin rotations on two individual electrons were realized by integrating a micrometre-size ferromagnet into a double-quantum-dot device. We find that it is the stray magnetic field of the micromagnet that enables the electrical control and spin selectivity. The results suggest that our approach can be tailored to multidot architecture and therefore could open an avenue towards manipulating electron spins electrically in a scalable way.

Magnetic resonance was recently used to coherently manipulate the spin of a single electron ${ }^{5}$ in a semiconductor structure, called a quantum $\operatorname{dot}^{8,9}$, whose tally of electrons can be tuned one by one, down to a single charge $\mathrm{e}^{10,11}$. However, producing strong and localized oscillating magnetic fields, which is a necessary step for addressing individual spins, is technically demanding. It involves on-chip coils ${ }^{5,12}$, relatively bulky to couple with a single spin, dissipating a significant amount of heat close to the electrons, whose temperature must not exceed a few decikelvins. In comparison, strong and local electric fields can be generated by simply exciting a tiny gate electrode nearby the target spin with low-level voltages. For scalability purposes, it is therefore highly desirable to manipulate electron spins with electric fields instead of magnetic fields.

To benefit from the advantages of electrical excitation, a mediating mechanism must be in place to couple the electric field to the electron spin, which usually responds only to magnetic fields. Spin-orbit coupling ${ }^{13,14}$, hyperfine interaction ${ }^{15}$ and $g$-factor modulation $^{16}$ work as the mediating mechanism, which attract interest for their physical origins but necessitate refinement in terms of both manipulation speed and scalability. Instead, we controllably mix the spin and charge degrees of freedom in a magnetic-field gradient ${ }^{6}$, very much like the Stern-Gerlach effect ${ }^{17}$. This allows for greater flexibility, because the method is applicable to any semiconductor material. In addition, the magnetic field profile can be engineered to enable the selective manipulation of several spins using a single electrode.

Thereby, we demonstrate addressable voltage-driven singlespin electron spin resonance (ESR) in a magnetic-field gradient. Two electrons are confined and spatially separated from each other in a gate-defined double quantum $\operatorname{dot}^{18}$ (Fig. 1a). The a.c. electric field, $E_{\text {a.c. }}$, is generated by exciting a nearby gate that couples to both spins (with presumably smaller strength for the right electron). The magnetic-field gradient is obtained by using a ferromagnetic strip that we integrate on top of the double-dot structure. The strip is magnetized uniformly along its hard axis by applying an in-plane magnetic field, $B_{0}$, stronger than the micromagnet's saturation field $(\sim 2 \mathrm{~T})$. In this condition, the resulting stray magnetic field has an out-of-plane component that varies linearly with position, pointing in the upward (or downward) direction to the left (or right) of the quantum-dot locations (Fig. 1b). In addition, the inhomogeneity of the in-plane component yields two different quantum-dot Zeeman fields $B_{0 \mathrm{~L}}$ and $B_{0 \mathrm{R}}$ (Fig. 1c). We use this feature to probe each spin separately.

To achieve ESR, we periodically displace the two electrons around their respective equilibrium positions in the slanting field. In each dot, the spin feels an upward magnetic field when the charge is displaced to the left. Conversely, the electron experiences a magnetic field pointing in the downward direction when displaced to the right. This effective oscillatory magnetic field induces transitions between the electron spin states (pointing in the direction parallel or antiparallel to the external field $B_{0}$ ) only when the driving frequency, $f$, matches the Larmor frequency, $f_{0}$, of the target spin. The latter is proportional to the corresponding quantum-dot Zeeman field $\left(h f_{0 \mathrm{~L}, \mathrm{R}}=g \mu_{\mathrm{B}} B_{0 \mathrm{~L}, \mathrm{R}}\right.$, where $h$ is the Planck constant, $g$ the Landé factor and $\mu_{\mathrm{B}}$ the Bohr magneton). By adjusting the frequency, phase and duration of the a.c. electric field burst used to periodically displace the electrons, arbitrary single spin rotations can then be realized in each dot through the ESR 

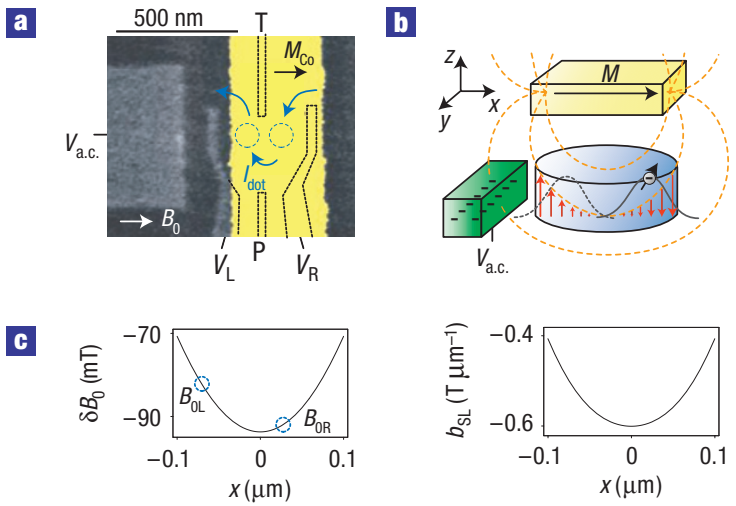

d

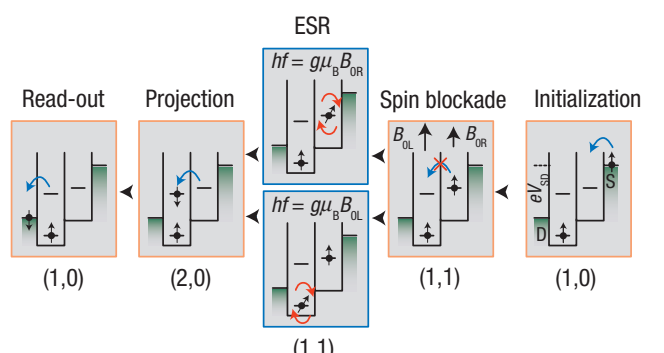

$\boldsymbol{e}$

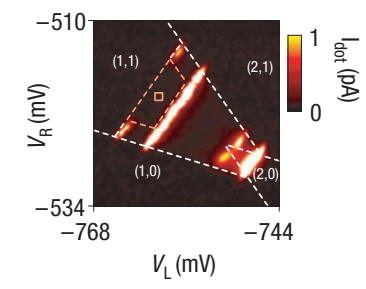

Figure 1 Device and read-out scheme. a, Scanning electron micrograph of a device similar to the one used in the experiment. The Ti-Au gates (light grey) are deposited on top of a GaAs-AIGaAs heterostructure containing a two-dimensional electron gas $90 \mathrm{~nm}$ below the surface. The $70-\mathrm{nm}$-thick cobalt micromagnet (artificially coloured in yellow) is isolated from the gate structure by an 80 -nm-thick calixarene insulating layer. Gates covered by the cobalt are outlined for clarity. The barriers separating the dots from each other and the reservoirs are tuned using the $P$ and $T$ gates. Blue arrows indicate the current flow through the two quantum dots (dotted circles) connected in series to source and drain. $\mathbf{b}$, The magnetization $\mathbf{M}$ (parallel to in-plane magnetic field $\mathbf{B}_{0}$ ) produces across the quantum dots (only one is shown in blue for clarity) a transverse stray magnetic field (red arrows) of the slanting form: $\mathbf{B}_{z}=b_{\mathrm{SL}} x \hat{z}$. The periodical displacement of the electron's wavefunction is driven by the a.c. gate voltage $V_{\text {a.c. }}$. c, Simulated profiles of the in-plane field shift, $\delta B_{0}=B_{x}-B_{0}$, and gradient of the transverse stray field, $b_{\mathrm{SL}}=\partial_{x} B_{z}$, near the double-dot location. The origin is taken at the centre of the micromagnet. For slight misalignment, the in-plane field produces two different quantum-dot Zeeman fields $B_{0 \mathrm{~L}}$ and $B_{0 \mathrm{R}}$. d, The sequential flow of electrons at spin blockade. This cycle can be described via the occupations $\left(N_{\mathrm{L}}, N_{\mathrm{R}}\right)$ of the left and right dots as $(1,0) \rightarrow(1,1) \rightarrow(2,0) \rightarrow(1,0)$. Starting from $(1,0)$, an electron tunnels from the source to form the $(1,1) \mathrm{T}_{+}$triplet state. The electron in the right dot cannot tunnel to the left dot because of the Pauli exclusion principle and transport is blocked. With ESR, the electron on the right dot can tunnel to the left dot to form the $(2,0)$ state. One of the two electrons then tunnels out to the drain to complete the cycle, yielding a finite leakage current. e, Stability diagram measured as a function of gate voltages $V_{L}$ and $V_{R}$ with $V_{\text {a.c. }}=0$ for source-drain bias $V_{\mathrm{SD}}=1.4 \mathrm{mV}$ at $B_{0}=2 \mathrm{~T}$. In the regions marked by $\left(N_{\mathrm{L}}, N_{\mathrm{R}}\right)$, transport is quenched owing to Coulomb blockade where each dot holds a fixed number of electrons $N_{\mathrm{L}}$ and $N_{R}$. Coulomb blockade is lifted in the triangular regions, where current flows except for the spin blockade area (enclosed by orange dotted lines).
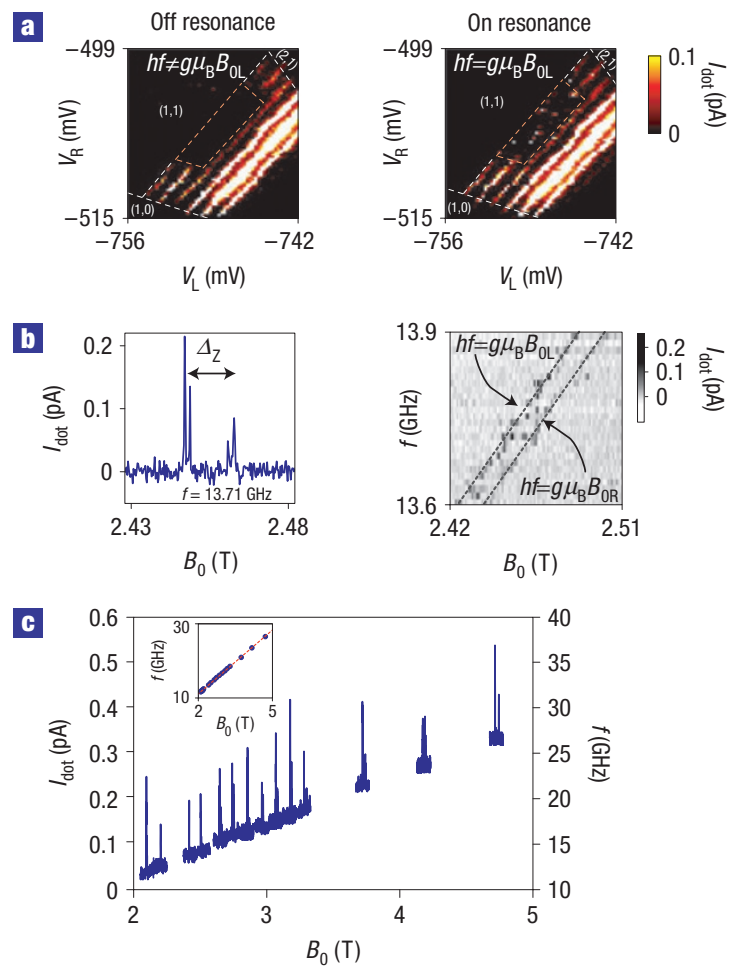

Figure 2 Electrically driven single-spin resonance. a, Stability diagrams under continuous electric excitation taken at frequency $f=26.5 \mathrm{GHz}$ and a power level of $-38 \mathrm{dBm}$. In the left (right) colour plots, the Larmor frequency in the left dot is detuned (tuned) to $f$ using the external magnetic field $\left(B_{0}=4.7 \mathrm{~T}\right.$ and $4.712 \mathrm{~T}$ respectively). $\mathbf{b}$, (left) Dot current as a function of $B_{0}$ measured at frequency and magnetic field half those in a after adjusting $V_{L}$ and $V_{R}$ to a resonant point of the spin-blockade region. (right) Similar scans obtained over a small range of frequencies. The dashed lines are guides to the eye to indicate the linear dependency of the two ESR peaks associated with the left and right dots. The power level is adjusted at each frequency to keep $E_{\text {a.c. }}$ constant. c, The two ESR peaks obtained over a wide range of magnetic field. Each trace is offset by an amount proportional to the corresponding frequency. Inset: Position of the left-dot peak. Each data point is obtained by averaging the peak position over five magnetic-field sweeps. Error bars are smaller than the symbols. The dashed red line is a linear fit, yielding $g=0.41 \pm 0.01$.

effect, a prerequisite for realizing the CNOT gate using the exchange interaction between neighbouring spins ${ }^{19}$.

To detect the electrically induced spin flips, we operate the double dot in the Pauli spin-blockade regime, where no current flows unless spin flips occur in either dot ${ }^{5,20}$ (Fig. 1d). The blockade arises because of Pauli exclusion: once the electron in the right dot forms a spin-polarized triplet with the electron in the left dot (either the $\mathrm{T}_{+}=\uparrow \uparrow$ or $\mathrm{T}_{-}=\downarrow \downarrow$ state), the right electron cannot move to the left dot. The spin-blockade regime is identified in the double-dot stability diagram by mapping the dot current, $I_{\mathrm{dot}}$, under large source-drain bias as a function of the left and right quantum dot gate voltages (Fig. 1e).

We now show that electric excitation can induce single-electron spin flips. We apply a continuous microwave voltage and follow the stability diagram around the resonance condition (Fig. 2a). The resulting electric field modifies the diagram through a process known as photon-assisted tunnelling ${ }^{21}$ (PAT). In general, PAT can assist electrons in breaking the spin blockade by, for instance, hopping to the left dot triplet states, usually energetically 


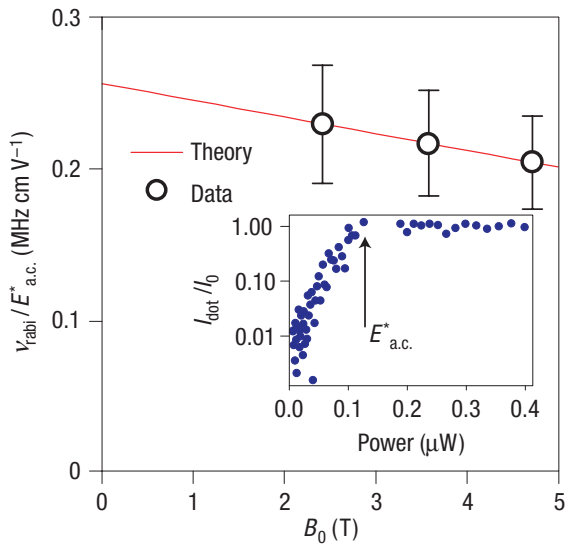

Figure 3 Dependency of the Rabi frequency on external field. Each data point is obtained after carrying out a power dependency of the ESR peak height (associated with the left dot) to extract the value of the electric field at saturation, $E_{\mathrm{acc}}^{*}$, and corresponding $B_{\text {a.c. }}$. The error bars are given by $\nu_{\text {Rabi }} / E_{\text {a.c. }}^{*} \cdot \delta E / E_{\text {a.c. }}^{*}$, where $\delta E$ is the error in the electric-field amplitude. The inset shows such power dependency obtained at $B_{0}=2.14 \mathrm{~T}$. The dot current is normalized over the saturation current $I_{0}$, which also depends on power owing to the PAT process (see Supplementary Information, Fig. S2). The red line is a fit to equation (1) assuming harmonic confinement with estimated energy $\hbar \omega_{0} \sim 1 \mathrm{meV}\left(\Delta=\hbar \omega_{0}, l_{\text {orb }}=\sqrt{\hbar / m^{*} \omega_{0}}\right.$, where $m^{*}$ is the effective electron mass, equal to 0.067 times the free-electron mass) and $g=0.41$.

inaccessible. For our power level (typically $-40 \mathrm{dBm}$ taking into account line attenuation), a clear spin-blockade region remains with leakage current below the noise floor of the experiment (20 fA). The spin blockade is lifted off by PAT only at much higher power level ( $-20 \mathrm{dBm}, 100 \mathrm{fA}$ leakage current).

The situation is different at the resonance condition for ESR, where a finite leakage current now flows in the spin-blockaded region. Starting from the $\uparrow \uparrow$ state, an ESR field resonant with the left (right) electron changes the initial state to the $\downarrow \uparrow(\uparrow \downarrow)$ configuration. Expressed in the singlet-triplet measurement basis ( $\left.\mathrm{S}, \mathrm{T}_{0}, \mathrm{~T}_{ \pm}\right), \downarrow \uparrow$ or $\uparrow \downarrow$ is an equal superposition of the $\mathrm{T}_{0}$ and $\mathrm{S}$ states. For the singlet component (S) of this state, the right electron can tunnel immediately to the left dot because the left dot singlet state is energetically accessible. The $\mathrm{T}_{0}$ component first evolves into $\mathrm{S}$ (owing to the large difference in quantum-dot Zeeman fields, $\Delta_{\mathrm{Z}}$, compared with the exchange energy $J$, that is, $g \mu_{\mathrm{B}} \Delta_{\mathrm{Z}} \gg J$ ), and then the right electron can move to the left dot as well ${ }^{22}$. Thus, by resonantly flipping the spin of the electron residing on either dot to form an antiparallel spin state, an electron charge moves through the dots, thereby lifting the spin blockade. The resonant response is observed clearly as $B_{0}$ and $f$ are varied for constant $E_{\text {a.c. }}$ (Fig. 2b). Two equally spaced peaks (with spacing $\Delta_{\mathrm{Z}}=13 \pm 2 \mathrm{mT}$ ) in $I_{\mathrm{dot}}$ are seen at a frequency proportional to $B_{0}$. Judging from the amplitudes, we attribute the first (second) peak to spin flips of the electron residing on the left (right) dot. This selective addressing is enabled by the inhomogeneous in-plane stray-field profile mentioned above. By slightly modifying the micromagnet geometry, the frequency selectivity can, in principle, be used to address individual spins in a scalable way (see Supplementary Information, Note A.1).

The linear dependency of each resonance on the external magnetic field is a key signature of ESR because the Larmor frequency is proportional to $B_{0}$. From the averaged position of one of the ESR peaks obtained over a wider range of magnetic field (Fig. 2c), we determine $g=0.41 \pm 0.01$, in good agreement for our type of device. Following the peak position below the micromagnet's saturation field, we have confirmed that $B_{0 \mathrm{~L}, \mathrm{R}}$ are smaller than the external field, a feature expected for the stray magnetic field (see Supplementary Information, Fig. S1a).

Evidence for spin-charge coupling induced by the slanting field is revealed in the ESR peak height. This gives information on the effective a.c. magnetic-field strength, $B_{\text {a.c. }}$, which is proportional not only to $E_{\text {a.c. }}$ but also to the magnetic-field gradient, $b_{\mathrm{SL}}$. To estimate $B_{\text {a.c. }}$, we use the non-monotonic response of the peak height to microwave power. As the power level is raised, the peak amplitude initially increases and then saturates past a certain level corresponding to an electric field $E^{*}$ (Fig. 3, inset). This response results from the interplay between the ESR and fluctuating Overhauser fields. The Overhauser field arises from hyperfine interaction between the electron and nucleus spins of the host material ${ }^{23}$. This interaction shifts the Larmor frequency randomly by an amount $\Delta f_{0}=g \mu_{\mathrm{B}} B_{\mathrm{N}} / h$, where $B_{\mathrm{N}}$ is the amplitude of the nuclear field fluctuations. For $B_{\text {a.c. }}>B_{\mathrm{N}}$, power broadening washes out the fluctuations. Every time an electron blocks the transport by spin blockade, the ESR field flips its spin and the current flow is therefore saturated. For $B_{\text {a.c. }}<B_{\mathrm{N}}$ the resonance condition is met only occasionally. Fewer electron spins are flipped per unit time and the current is consequently lower. Saturation occurs at $B_{\text {a.c. }} \sim B_{\mathrm{N}} / 2$ (refs 15,22).

The Overhauser field fluctuations are also responsible for the jitter in the peak position visible in Fig. $2 b$, which enables us to extract $B_{\mathrm{N}}=2.4 \mathrm{mT}$. Using this result, we estimate $B_{\text {a.c. }}$ to be $1 \mathrm{mT}$ at the onset of saturation. Remarkably, such a magnitude is obtained for microwave power 500 times smaller than for magnetically driven ESR with an on-chip coil ${ }^{5,12}$. By operating deeper in the Coulomb-blockade region of the stability diagram, fields as strong as $10 \mathrm{mT}$ are possible because stronger PAT is required to lift the spin blockade, yielding a spin-flip time as fast as $20 \mathrm{~ns}$. The efficiency can further be improved by increasing the micromagnet thickness and using stronger ferromagnetic materials ${ }^{7}$.

In Fig. 3, we plot the estimated spin-flip rate (Rabi frequency, $\left.v_{\text {Rabi }}=g \mu_{\mathrm{B}} B_{\text {a.c. }} / 2 h\right)$ normalized over electric field in a magnetic-field range above the micromagnet saturation field. The normalized Rabi frequency does not vary significantly with $B_{0}$, as expected because $b_{\mathrm{SL}}$ should be constant in this regime. A linear fit through the data suggests that a second field also contributes, on a smaller level, to the effective ESR field. We attribute the second contribution to the usual spin-orbit coupling present in most semiconductors. The latter gives rise to an intrinsic slanting magnetic field of slope $2 B_{0} / l_{\mathrm{so}}$, where $l_{\mathrm{so}}$ is the characteristic spin-orbit length ${ }^{13,24}$. The precise profile of this effective magnetic field depends on the relative orientation of $B_{0}$ and $E_{\text {a.c. }}$ with respect to the crystal directions. For our geometry, the spin-orbit contribution works in reducing the ESR field when $l_{\text {so }}>0$, a trend observed in the data. To be more quantitative, we derived the following expression for the total effective ESR field strength (see Supplementary Information, Note A.2):

$$
B_{\text {a.c. }}=\frac{e E_{\text {a.c. }} l_{\text {orb }}}{\Delta}\left(\left|b_{\mathrm{SL}}\right|-\frac{2\left|B_{0}\right|}{l_{\text {so }}}\right) l_{\text {orb }},
$$

where $\Delta$ and $l_{\text {orb }}$ are the quantum dot's confinement energy and orbital spread. The fit to equation (1) yields $\left|b_{\mathrm{SL}}\right| \sim 0.8 \mathrm{~T} \mu \mathrm{m}^{-1}$, in good agreement with the expected stray-magnetic-field profile, and $l_{\text {so }} \sim 58 \mu \mathrm{m}$, whose magnitude is consistent with recently observed spin-orbit-mediated ESR in a similar system ${ }^{13}$. The fluctuating nuclei field was shown to also enable electrically driven spin flips ${ }^{15}$, and should, like the spin-orbit case, contribute to our ESR 
signal. However, the weak ESR response observed at low external magnetic fields (where $b_{\mathrm{SL}} \sim 0$ ) implies that the hyperfine effect does not contribute significantly to the effective ESR field (see Supplementary Information, Fig. S1b).

The spin rotations demonstrated here, in combination with experimentally realized spin read-out ${ }^{25,26}$ and tunable exchange coupling ${ }^{27,28}$, fulfil many of the requirements for quantum computing with electron spins in quantum dots ${ }^{19}$ using only electric fields. In contrast to previously reported voltage-driven ESR mediated by spin-orbit coupling or interaction with nucleus spins, our scheme, which is applicable to any material, does not rely on intrinsic properties that are also responsible for degrading the spin coherence in solid-state systems. The coherence time for our hybridized spin is expected to be as long as $1 \mathrm{~ms}$ using cleaner materials ${ }^{6}$ such as carbon nanotubes, Si nanowires and SiGe heterostructures. Moreover, micromagnets may simplify the daunting task of integrating many quantum dots into a multiqubit quantum register. The independent addressing of the spin in each of the double dots observed here and in ref. 15 inspires scalability with the help of micromagnets. By engineering the stray-field profile, a common ESR gate could be used to operate on any spin in the register, simply by matching the driving frequency to a position-dependent Zeeman field.

\section{METHODS}

The measurements were made in an Oxford Instrument Kelvinox 100 dilution refrigerator operating at a base temperature of $40 \mathrm{mK}$ with an estimated electronic temperature of $200 \mathrm{mK}$. The microwave signal is applied to the ESR gate using a commercial microwave source (Agilent $8360 \mathrm{~B}$ ). The ESR gate is part of an on-chip coplanar waveguide, which is wire bonded to the sample holder's alumina coplanar waveguide. The latter is connected to the high-frequency line of the dilution refrigerator using a microwave bead. These precautions are taken to minimize loss by improving impedance mismatches.

The GaAs-AlGas heterostructure from which the sample was made was purchased from Sumitomo Electric. The two-dimensional electron gas has a mobility of $1 \times 10^{2} \mathrm{~m}^{2} \mathrm{~V}^{-1} \mathrm{~s}^{-1}$ and an electron density of $3 \times 10^{15} \mathrm{~m}^{-2}$ measured at $1.4 \mathrm{~K}$. The device used in this experiment suffered initially from a high level of telegraphic noise, associated with the switching of a few background charges. This made the quantum-dot behaviour extremely unstable. To improve the charge stability, the device was first cooled down under a positive bias of $+0.5 \mathrm{~V}$ applied to the quantum dot gates. A negative bias of $-2 \mathrm{~V}$ was then applied to the micromagnet once the device had reached the base temperature.

The occupation of the double dot by only two electrons was confirmed by first opening the interdot barrier (using the $\mathrm{P}$ gate) to form a single dot and then opening the barriers separating the dot from the source and drain reservoirs (with the T gate). Under these conditions, no extra Coulomb-blockade peaks appeared in the region $N_{\mathrm{L}}+N_{\mathrm{R}}=0$ of the double-dot stability diagram.

The stray magnetic field produced by the ferromagnetic strip is calculated numerically using the Mathematica package Radia, available at http://www.esrf.fr, assuming a uniform magnetization and using the saturation magnetization of cobalt $\left(\mu_{0} M_{\mathrm{Co}}=1.8 \mathrm{~T}\right)$. The strip is $5 \mu \mathrm{m}$ long.

Received 18 January 2008; accepted 7 July 2008; published 17 August 2008.
References

1. Awschalom, D., Loss, D. \& Samarth, N. Semiconductor Spintronics and Quantum Computation (Springer, Berlin, 2002).

2. Poole, C. Electron Spin Resonance 2nd edn (Wiley, New York, 1993).

3. Xiao, M., Martin, I., Yablonovitch, E. \& Jiang, H. W. Electrical detection of the spin resonance of a single electron in a silicon field-effect transistor. Nature 430, 435-439 (2004).

4. Jelezko, F., Gaebel, T., Popa, I., Gruber, A. \& Wrachtrup, J. Observation of coherent oscillations in a single electron spin. Phys. Rev. Lett. 92, 076401 (2004).

5. Koppens, F. H. L. et al. Driven coherent oscillations of a single electron spin in a quantum dot. Nature 442, 766-771 (2006).

6. Tokura, Y., Van der Wiel, W. G., Obata, T. \& Tarucha, S. Coherent single electron spin control in a slanting Zeeman field. Phys. Rev. Lett. 96, 047202 (2006).

7. Pioro-Ladrière, M., Tokura, Y., Obata, T., Kubo, T. \& Tarucha, S. Micromagnets for coherent control of spin-charge qubit in lateral quantum dots. Appl. Phys. Lett. 90, 024105 (2007).

8. Kouwenhoven, L. P. \& Marcus, C. Quantum dots. Phys. World 11, 35-39 (June 1998).

9. Jacak, L., Hawrylak, P. \& Wojs, A. Quantum Dots (Springer, Berlin, 1998).

10. Tarucha, S., Austing, D. G., Honda, T., van der Hage, R. J. \& Kouwenhoven, L. P. Shell filling and spin effects in a few electron quantum dot. Phys. Rev. Lett. 77, 3613-3616 (1996).

11. Ciorga, M. et al. Addition sprectrum of a lateral dot from Coulomb and spin-blockade spectroscopy. Phys. Rev. B 61, R16315 (2000).

12. Obata, T. et al. Microwave band on-chip coil technique for single electron spin resonance in a quantum dot. Rev. Sci. Instrum. 78, 104704 (2007).

13. Nowack, K. C., Koppens, F. H. L., Nazarov, Y. V. \& Vendersypen, L. M. K. Coherent control of a single spin with electric fields. Science 318, 1430-1433 (2007).

14. Meir, L. et al. Measurement of Rashba and Dresselhaus spin-orbit magnetic fields. Nature Phys. 3 , 650-654 (2007).

15. Laird, E. A. et al. Hyperfine-mediated gate-driven electron spin resonance. Phys. Rev. Lett. 99, 246601 (2007).

16. Kato, Y. et al. Gigahertz electron spin manipulation using voltage-controlled g-tensor modulation Science 299, 1201-1204 (2003).

17. Gerlach, W. \& Stern, O. Der experimentelle nachweiss der richtungsquantelung im magnetfeld. Z. Phys. 9, 349-355 (1922).

18. Hüttel, A. K., Ludwig, S., Lorenz, H., Eberl, K. \& Kotthaus, J. P. Direct control of the tunnel splitting in a one-electron double quantum dot. Phys. Rev. B 72, 081310(R) (2005).

19. Loss, D. \& DiVicenzo, D. P. Quantum computation with quantum dots. Phys. Rev. A 57, $120-126(1998)$.

20. Ono, K., Austing, D. G., Tokura, Y. \& Tarucha, S. Current rectification by Pauli exclusion in a weakly coupled double quantum dot system. Science 297, 1313-1317 (2002).

21. van der Wiel, W. G. et al. Electron transport through double quantum dots. Rev. Mod. Phys. 75 $1-22$ (2003).

22. Koppens, F. H. L. et al. Detection of single electron spin resonance in a double quantum dot. J. Appl. Phys. 101, 081706 (2007).

23. Coish, W. A \& Loss, D. Hyperfine interaction in a quantum dot: Non-Markovian electron spin dynamics. Phys. Rev. B 70, 195340 (2004).

24. Golovach, V. N., Borhani, M. \& Loss, D. Electric-dipole-induced spin resonance in quantum dots. Phys. Rev. B 74, 165319 (2006).

25. Ciorga, M. et al. Collapse of the spin-singlet phase in quantum dots. Phys. Rev. Lett. 88 256804 (2002).

26. Elzerman, J. M. et al. Single-shot read-out of an individual electron spin in a quantum dot. Nature 430, 431-435 (2004).

27. Petta, J. R. et al. Coherent manipulation of coupled electron spins in semiconductor quantum dots. Science 309, 2180-2184 (2005).

28. Hatano, T., Stopa, M. \& Tarucha, S. Single-electron delocalization in hybrid vertical-lateral double quantum dots. Science 309, 268-271 (2005).

Supplementary Information accompanies the paper at www.nature.com/naturephysics.

\section{Acknowledgements}

We thank F. H. L. Koppens and C. Buizert for discussions, I. Mahboob for comments and Y. Sekine for advice. S.T. acknowledges financial support from Grants-in-Aid for Scientific Research S

(No 19104007) and B (No 18340081).

\section{Author contributions}

M.P.-L. designed the experiment, fabricated the device and wrote the paper. T.O. ensured proper operation of microwaves. M.P.-L. and T.O. carried out the bulk of the experimental work and analysis. Y.T. conceived the theory. Y.-S.S. participated in experiments. T.K. participated in the theoretical work. K.Y. assisted with device processing. T.T. assisted with micromagnet technology. S.T. planned the project. All authors discussed the results and commented on the manuscript.

\section{Author information}

Reprints and permission information is available online at http://npg.nature.com/reprintsandpermissions. Correspondence and requests for materials should be addressed to M.P.-L. 